\title{
BMJ Open Protocol to test the efficacy and safety of frequent applications of skin electrical stimulation for Leber hereditary optic neuropathy: a single-arm, open-label, non-randomised prospective study
}

Kaori Ueda (1) , Takuji Kurimoto, Fumio Takano, Yusuke Murai, Sotaro Mori, Mari Sakamoto, Takayuki Nagai, Yuko Yamada-Nakanishi, Makoto Nakamura

To cite: Ueda K, Kurimoto T, Takano F, et al. Protocol to test the efficacy and safety of frequent applications of skin electrical stimulation for Leber hereditary optic neuropathy: a single-arm, open-label, non-randomised prospective study. BMJ Open 2021;11:e048814. doi:10.1136/ bmjopen-2021-048814

- Prepublication history for this paper is available online To view these files, please visit the journal online (http://dx.doi. org/10.1136/bmjopen-2021048814).

Received 07 January 2021 Accepted 04 October 2021
Check for updates

(c) Author(s) (or their employer(s)) 2021. Re-use permitted under CC BY-NC. No commercial re-use. See rights and permissions. Published by BMJ.

Division of Ophthalmology, Department of Surgery, Kobe University Graduate School of Medicine, Kobe, Japan

Correspondence to Dr Takuji Kurimoto; kuritaku12011201@yahoo.co.jp

\section{ABSTRACT}

Introduction Leber hereditary optic neuropathy (LHON) is an acute or subacute inherited optic neuropathy caused by mitochondrial mutations. More than $90 \%$ of patients with LHON have one of three point mutations (ie, G3460A, G11778A and T14484C). We previously reported that a 12week session of skin electrical stimulation (SES) with a 2week interval significantly improved visual acuity and field tests 1 week after the last stimulation and without adverse effects in 10 cases of LHON carrying the mt DNA G11778A mutation. In the present study, we will examine the magnitude and persistence of the efficacy and presence or absence of adverse events using SES with a more frequent stimulation protocol.

Methods and analysis This study will be a single-arm, open-labelled, non-randomised clinical study that analyses 15 cases of LHON with G11778A mutation. All participants will take a portable SES device home and perform SES by themselves every other day for 12 weeks. The logarithm for the minimum angle of resolution (logMAR) bestcorrected visual acuity (BCVA) at 1 week after the last SES will be measured as the primary outcome. LogMAR BCVA will be measured at four and 8 weeks after the last SES treatment. The Humphrey visual field sensitivity test using size $V$ stimulation and critical fusion frequency at 1, 4 and 8 weeks after the last SES session will be secondary outcome measurements. Slit-lamp examination, optical coherence tomography and specular microscopy will also be performed to verify the safety of SES.

Ethics and dissemination The protocol was approved by the Institutional Review Board at Kobe University, Japan (Approval No.C190030). This study is in progress and deserves Pre-result. All documents communicating with the ethics committee will be reposited by the researcher. Modifications to the protocol will be reviewed by the ethics committee and implemented after approval. Data monitoring will be performed by a researcher who is not involved in the study every 6 months after approval. The research summary results will be registered in the Japan Registry of Clinical Trials (jRCTs) and made available to participants in accordance with the terms described in the documents. In addition, the results of this study will be presented at domestic and international meetings and

\section{Strengths and limitations of this study}

- The first protocol applied to frequent electrical stimulation using a portable electrical stimulation device for intractable optic neuropathy Leber hereditary optic neuropathy patients.

- Feasibility of extending the adaptation of skin electrical stimulation as a treatment for other intractable optic neuropathies, including ischaemic optic neuropathy, traumatic optic neuropathy and normaltension glaucoma.

- Although this study will not be controlled, the results will provide invaluable information regarding the feasibility of conducting a large-scale randomised trial.

published in peer-reviewed journals within a year after data is fixed.

Trial registration number jRCTs052200033.

\section{INTRODUCTION}

Leber hereditary optic neuropathy (LHON) is a maternally inherited, acute or subacute optic neuropathy that is caused by point mutations in mitochondrial DNA. One of three mitochondrial gene mutations at positions mt3460, mt11778 and mt14484 have been identified in $>95 \%$ of LHON patients. ${ }^{1}$ In particular, the overwhelming majority $(80 \%)$ of Japanese patients with LHON possess the mt11778 mutation. Traditionally, LHON primarily affects men from adolescence to their thirties. However, we recently conducted a domestic nationwide survey which revealed that the number of elderly patients with LHON has increased over time. ${ }^{2}$ As for treatment, several clinical trials have been conducted, including one assessing the oral medication, idebenone, a short-chain benzoquinone of coenzyme Q10, ${ }^{3}$ and a gene 
therapy study using an adeno-associated virus (AAV) vector. ${ }^{4}$ Unfortunately, all clinical trials failed to show a significant effect on patients with respect to the recovery of visual function.

Several basic studies demonstrated that electrical stimulation (ES) to the eye has survival-promoting effects on degenerated retinal photoreceptor cells or retinal ganglion cells (RGCs) ${ }^{5-7}$ Based on these lines of evidence, a variety of ES delivery methods to the eyes, including transcorneal, transpalpebral, transorbital and transcranial approaches, have been applied for intractable retinal and optic nerve diseases. ${ }^{8-12}$ Recently, we have applied skin ES (SES), usually classified as transorbital ES, to 10 cases of LHON harbouring a G11778A mutation. ${ }^{13} 14$ The short-term results from six consecutive SES sessions every 2 weeks indicated a significant best-corrected visual acuity (BCVA), and half of the subjects experienced a gradual improvement in visual field sensitivities until 2 months following the last SES session. In addition, there were no adverse effects associated with SES, such as skin issues resulting from the attached electrode gel pads.

Previous studies also provided important evidence suggesting that more frequent ES sessions may augment the survival-promoting effects. ${ }^{15}{ }^{16}$ Morimoto et al revealed that four consecutive transcorneal ES sessions every 3-4 days significantly increased RGC survival rate compared with a single stimulation. ${ }^{16}$ Tagami et al also reported that twelve consecutive ES sessions to crushed optic nerves of rats markedly increased the RGC survival rate compared with a single ES session. ${ }^{15}$ However, clinical trials have not been performed to determine whether frequent ES applications enhance the effects of ES in cases with intractable optic neuropathy and degenerative retinal diseases.

In the present study, we will determine whether more frequent SES sessions using a small portable ES-generating device augments the effects of SES for patients with LHON harbouring the G11778A mutation and whether more frequent SES sessions increase the adverse effects.

\section{METHODS AND ANALYSIS \\ Study design}

This will be a single-arm, non-randomised, exploratory prospective study to preliminarily test the efficacy and safety of frequent SES sessions for patients with LHON carrying an mtDNA G11778A mutation using a home healthcare medical device for 3 months. The protocol was approved by the Institutional Review Board at Kobe University, Japan (Approval No.C190030). The outline of this research protocol was based on our previous studies. ${ }^{1314}$

\section{Informed consent}

Fifteen patients with LHON carrying the mtDNA G11778A mutation will be enrolled. All participants will provide written informed consent and orally receive detailed protocol instructions. Informed consent will

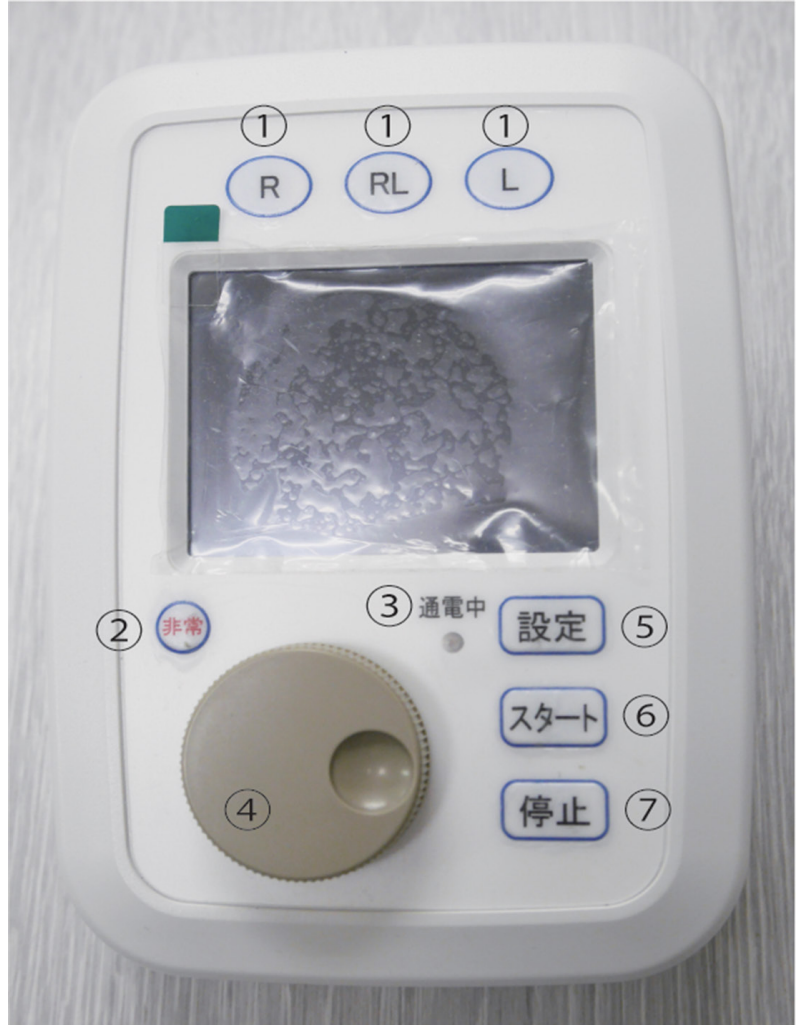

Figure 1 The portable device used for skin electrical stimulation. Each number indicates the components of the portable device. (1) the button for the selection of the stimulated eye: $R$, right eye, RL, both eyes, L, left eye. (2) The button for the emergency stop. (3) The power indicator. (4) Adjustment knob for the amount of current. (5) The menu button to manipulate the conditions of electrical stimulation. (6) The button to start electrical stimulation. (7) The pause button to temporarily stop electrical stimulation. If the button is hit once more, electrical stimulation will restart from the time point of the pause.

include the purpose of the study, a detailed schedule for the examinations, and a list of possible adverse effects or risks associated with the treatment. Participants will also be informed that they could withdraw from the study at any time for any reason and that there will be no disadvantages to future medical care. No patient will participate in the study without the formal procedure of informed consent.

\section{Intervention}

The participants well be given a small portable device produced by the Mayo to electrically stimulate their eyes by themselves at home. The size of the machine is $14 \times 10 \times 6 \mathrm{~cm}$ (figure 1 ). Two electrode pads are attached on the forehead above the target eye, that is, above the eyebrow and under the lower eyelid. After obtaining informed consent and before the participants bring the device home, they will receive detailed instructions from the investigators on how to use the instrument.

The SES protocol includes a biphasic square wave, 1 $\mathrm{mA}$ amplitude, $10 \mathrm{~ms}$ duration, and $20 \mathrm{~Hz}$ frequency stimulus that will be applied for 30 min every other day for 12 


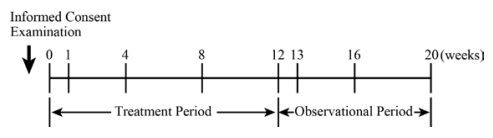

Figure 2 Study flow chart.

weeks. The current is set at a constant $1 \mathrm{~mA}$. The portable stimulator is programmed not to exceed a current intensity above $2 \mathrm{~mA}$. If the electrode pad become detached from the participant or a cable is disconnected from the stimulator, the current automatically ceases. When the stimulation time reaches $30 \mathrm{~min}$, the current will be automatically interrupted.

All participants will visit at 1, 4, 8 and 12 weeks after the initiation of SES (treatment period) and again at 1, 4 and 8 weeks after the last SES session (observational period; figure 2). With respect to ensuring safety, all SES sessions will be performed with the support of healthy individuals who have no visual problems. The SES conditions will be preset so that they cannot be modified. Each participant will receive a check sheet to confirm that they performed SES according to the schedule which will be returned on the day of each examination. When performing SES, the subjects will operate the device each time and check for abnormalities in the device to ensure safety.

\section{Inclusion and exclusion criteria}

The inclusion criteria are as follows: (1) age $\geq 16$ and $<80$ years, (2) diagnosis of LHON, (3) written informed consent from participants or legal representatives, (4) stable condition for $>8$ months after the onset of LHON, (5) BCVA $\geq 0.01$ and $<0.1$, (6) presence of mtDNA G11778A mutation and (7) ability to receive the support of individuals with normal vision during the SES sessions. The rationale for each inclusion criterion is as follows: (1) the susceptible age of LHON ranges from teenage years to approximately 30 years, although the number of middle to older age patients with LHON have increased recently; (2) patients with LHON containing an mtG11778A mutation have the lowest rate of spontaneous recovery ${ }^{1}$; (3) as described in the 'informed consent' section, all participants will receive the written informed consent sheet and detailed protocol instructions will be provided orally; (4) the LHON condition is thought to be stable after $>8$ months $^{17}$; and (5) in most patients, BCVA is $<0.1$. In patients with LHON with a BCVA $<0.01$, a therapeutic effect cannot be expected. ${ }^{14}$

The exclusion criteria are as follows: (1) BCVA $<0.01$; (2) smoking history within half a year before the initiation of the study; (3) use of electronic devices, such as a pacemaker; (4) history of intraocular surgery within a year; (5) ongoing treatment for any ocular disease; (6) history of idebenone treatment within a year; (7) ongoing treatment with either ethambutol, chloramphenicol, linezolid, erythromycin, streptomycin, antiretroviral drugs, amiodarone, infliximab, clioquinol, dapsone, quinine, pheniprazine, suramin sodium or isoniazid; (8) history of epilepsy; (9) pregnancy; (10) severe dermatitis; (11) participation in other clinical studies and (12) inappropriate cases as judged by physicians who are responsible for the study. The rationale for each criterion is as follows: (1) as for patients with LHON with a BCVA $<0.01$, a therapeutic effect cannot be expected; (2) smoking is considered an environmental factor in the onset and progression of LHON; (3) and (8) the instruments could affect other electronic medical devices or induce epileptic stroke; (4) and (5) intraocular surgery or other eye diseases affect visual function; (6) and (7) idebenone or other medications affect the optic nerve, especially idebenone, which has been reported to improve visual field sensitivities of patients with LHON; (9) the effect of SES on a foetus is unknown; (10) gel pads for ES adversely affect the skin; and (11) and (12) these are established from an ethical point of view.

\section{Sample size}

Fifteen patients with LHON carrying an mtDNA G11778A mutation will participate in the study. In our previous study, the mean value of logarithm for the minimum angle of resolution (logMAR) BCVA was 1.70 in seven patients with LHON and the inclusion criteria were consistent with those of the present study. The participants will receive SES once every 2 weeks for a total of six treatments. The treatment will improve to -0.10 (SD was $0.10)$ compared with the baseline value. Regarding the change in logMAR BCVA before and after SES sessions in the present study, we assume the average value of the difference will be -0.10 and the SD will be 0.10 based on our previous study. This indicates that the difference in the number of changes in logMAR BCVA between the present and previous studies will be -0.1 or less as augmentative effects of SES result from an increase in the number of SES applications. To secure a detection power of $80 \%$ when performing a paired Student's t-test with a onesided 5\% significance level employing the null hypothesis, the average difference in logMAR BCVA between baseline and 1 week after the last session SES should be -0.10 , thus, the study will require 10 cases. Considering some withdrawals after participation, we determined that the final required number of participants should be 15 .

\section{Parameters}

The data collected for statistical analysis will be as follows: sex, age, BCVA, critical fusion frequency (CFF) values and Humphrey visual field sensitivities programmed with 30-2, size V stimulation (Carl Zeiss Meditec, Dublin, California, USA). The thicknesses of the circumpapillary retinal nerve fibre layer (RNFL) and ganglion cell complex (GCC) will be measured by spectral-domain optic coherence tomography (Cirrus HD-OCT, Carl Zeiss, Jena, Germanny), and the cell density of the corneal endothelial cells will be measured by specular microscopy (Konan Medical, Nishinomiya, Japan).

\section{Outcome measures}

Table 1 shows the examination schedule in this study. Referring to our previous study, we will perform 
Table 1 Examination schedule

\begin{tabular}{|c|c|c|c|c|c|c|c|c|c|}
\hline & \multirow[b]{2}{*}{ Screening } & \multicolumn{5}{|c|}{ SES approach } & \multicolumn{3}{|c|}{ Observation } \\
\hline & & 0 week & 1 week & 4 weeks & 8 weeks & 12 weeks & 13 weeks & 16 weeks & 20 weeks \\
\hline Visit no & 1 & 2 & 3 & 4 & 5 & 6 & 7 & 8 & 9 \\
\hline \multicolumn{10}{|l|}{ SES approach } \\
\hline Informed consent & $\bullet$ & & & & & & & & \\
\hline Slit-lamp microscopy & $\bullet$ & & $\bullet$ & $\bullet$ & $\bullet$ & $\bullet$ & $\bullet$ & $\bullet$ & $\bullet$ \\
\hline Intraocular pressure & $\bullet$ & & $\bullet$ & $\bullet$ & $\bullet$ & $\bullet$ & $\bullet$ & $\bullet$ & $\bullet$ \\
\hline CFF & - & & - & $\bullet$ & $\bullet$ & $\bullet$ & 0 & $\bullet$ & $\bullet$ \\
\hline Specular microscope & - & & - & & & • & & & ○ \\
\hline Adverse effect review & & & - & - & - & - & - & - & $\bullet$ \\
\hline
\end{tabular}

BVCA, best-corrected visual acuity; CFF, critical fusion frequency; HVF, humphrey visual field; LogMAR, logarithm of the minimum angle of resolution; OCT, optical coherence tomography; SES, skin electrical stimulation.

examinations at baseline and eight visits during and after the SES sessions. The first examination will be scheduled prior to the initiation of SES as a baseline. The next four examinations will be performed at $1,4,8$ and 12 weeks during the treatment period. At the 1-week, 4-week and 8 -week visits, we will verify that the participants could correctly handle the instrument and electrode pads and conduct SES by themselves.

The last three examinations will be performed at 1,4 and 8 weeks after the last session of SES during the observation period. These examinations will include logMAR BCVA, HFA, CFF and OCT. Slit-lamp microscopy will also be performed at the same time to exclude the occurrence of unexpected adverse effects.

The primary outcome is defined as the difference in $\log$ MAR BCVA between baseline and 1 week after the final session of SES. Based on our previous study, ${ }^{14}$ the null hypothesis is the mean difference in logMAR BCVA at baseline and 1 week after the last session of SES $(-0.10)$ as analysed using a one-sample t-test with a two-sided significance level of $5 \%$. Rejection of the null hypothesis indicates that the tested frequent SES protocol was judged to be superior to that of the previous SES protocol.

The secondary outcomes will be defined as the difference in the logMAR BCVA at baseline and 4 or 8 weeks after the last session of SES. As for the visual field sensitivities measured by stimulation size $\mathrm{V}$, the summed sensitivity of 52 measurements, except for the outermost points, will be analysed between baseline and 4 and 8 weeks after the last SES session. The difference in mean CFF at baseline and 4 and 8 weeks after the last SES session will also be statistically analysed. Both primary and secondary outcomes will be analysed between the groups with or without a history of SES.

\section{Safety and tolerability}

To verify the safety of the frequent SES protocol, we will observe dermal changes around the attached electrode pad, ocular surface and anterior chamber by slit-lamp microscopy, measure the thicknesses of the RNFL and GCC with OCT, and examine the cell densities of corneal endothelial cells by specular microscopy. The schedule of examinations is shown in table 1.

\section{Statistical analysis}

The primary and secondary outcomes, that is, visual acuity and field measurements, will be analysed using the full analysis set. Participants will complete all SES sessions and examinations. Normality will be tested with a Shapiro-Wilk test. In the case of no rejection of normality, primary and secondary outcome measures comparing data at nonrepeated test points will be analysed with a paired t-test. In the case of rejection of normality, a Wilcoxon signedrank test will be applied. In the case of no rejection of normality, repeated measures as secondary outcomes will be analysed with a mixed-effects model using the least squares distance to address all available post-baseline data and determine whether the differences from baseline to each measurement point are statistically significant. In cases of normality rejection, a Wilcoxon signed-rank test will be applied using the closed test procedure. The test of the difference between 4 weeks and baseline will be conducted only if the test between 8 weeks and the baseline is rejected. Regarding among-group comparisons 
with or without a history of SES for both primary and secondary outcomes, an unpaired t-test or Mann-Whitney $\mathrm{U}$ test will be used depending on the aforementioned normality. Continuous variables will be expressed as the mean $\pm \mathrm{SD}$ for a Gaussian distribution and medians (IQR) for non-Gaussian distributions, whereas categorical variables for the descriptive data will be expressed as frequencies and proportions. Unless stated otherwise, the results will be presented as two-sided $p$ values, with a $p<0.05$ considered statistically significant. All statistical analyses will be performed using IBM SPSS (SPSS,24.0) software.

\section{Patient and public involvement}

There is no patient involved in this protocol.

\section{DISCUSSION}

Although idebenone, EPI-743 (defined as 2-[(3R,6E,10E)3-hydroxy-3,7,11,15-tetramethyl-6,10,14-hexadecatrien-1yl]-3,5,6-trimethyl-2,5-cyclohexadiene-1,4-dione) or gene therapy using an AAV-2 vector has resulted in partial recovery of visual function, ${ }^{418}$ an effective treatment for LHON has not yet been established. Clinically, it is well known that spontaneous recovery sometimes occurs in patients with LHON carrying an mtDNA G11778A mutation, even after development of optic atrophy. From another perspective, it may be reasonable that LHON becomes treatable if the appropriate treatment methods are discovered.

Our previous study showed that less frequent SES sessions improved visual function in patients with LHON carrying mtDNA G11778A mutations without any adverse effects. ${ }^{14}$ This improvement was maintained even for a short-term period after the cessation of SES. The SES device used in the present study is downsized and made portable to enable participants administer treatment at home. More frequent SES applied every other day may augment the effect of improving visual function.

The treatment methods for visual impairment using ES have been extensively reviewed in previous reports. ${ }^{129-21}$ In addition, Perin et al previously reviewed and categorised ES therapies into four classes depending on the scale of the clinical trials. There were five types of stimulation methods and three stimulation pathways: transcorneal, transcranial and transpalpebral electric stimulation. Of these stimulation methods, it has been reported that repetitive transorbital alternating current stimulation is recommended, although high-frequency random noise stimulation and trans-cranial direct current stimulation may also be effective.

The target diseases are almost the same for all ES methods. Severe and irreversible visual dysfunction is ischaemic optic neuropathy or retinal degenerative disease, including retinitis pigmentosa and wet-type agerelated macular degeneration. In cases where improvement in visual function was obtained by ES, the effect was reported to last for several months without any side effects.
Our study will be a non-controlled clinical trial corresponding to the smallest class 4 category in this report. However, our study differs from other reports regarding ES programmes or methods. In this study, SES will be performed and the electrode pads are small and only attach above the eyebrow and lower eyelid. The stimulation frequency will be every other day for 12 weeks, which is also a characteristic of our study. More intensive use of home medical treatment could be even more beneficial to patients with LHON and possibly for patients with other intractable rare optic nerve diseases, such as ischaemic optic neuropathy or traumatic optic neuropathy, compared with less frequent treatment in a hospital. This will also motivate clinicians to plan future prospective randomised clinical trials to obtain confirmative evidence of the efficacy of frequent SES for the treatment of patients with LHON.

\section{ETHICS AND DISSEMINATION}

This study received approval from the Institutional Review Board at Kobe University, Japan (approval no.C190030). All documents communicating with the ethics committee will be reposited by the researcher. Modifications to the protocol will be reviewed by the ethics committee and implemented after approval. Data monitoring will be performed by a researcher who is not involved in the study every 6 months after approval. The research summary results will be registered in the jRCTs and made available to participants in accordance with the terms described in the documents. In addition, the results of this study will be presented at domestic and international meetings and published in peer-reviewed journals within a year after data is fixed.

Acknowledgements The authors thank Enago (www.enago.jp) for the English language review.

Contributors KU and TK contributed to the study concept. KU, TK, YM, SM and MS were consulted for the statistical analysis for this study and developed analytic plan. KU and TK drafted the first manuscript and MS, TK, KU and MN revised the manuscript and completed the final manuscript. MN supervised the trial. KU, TK, FT, YM, TN, YY-N and MN made significant contributions to the concept and substantial design of the trial. All authors gave final approval of the version to be submitted and agree to be accountable for all aspects of the work.

Funding The authors have not declared a specific grant for this research from any funding agency in the public, commercial or not-for-profit sectors.

Competing interests None declared.

Patient and public involvement Patients and/or the public were not involved in the design, or conduct, or reporting, or dissemination plans of this research.

Patient consent for publication Not applicable.

Provenance and peer review Not commissioned; externally peer reviewed.

Data availability statement Correspondence: Takuji Kurimoto, M.D., Ph.D.Division of Ophthalmology, Department of SurgeryKobe University Graduate School of Medicine7-5-2 Kusunoki-cho, Chuo-ku, Kobe 650-0017, JapanPhone: 81-78381-6048; FAX: 81-78-382-6059E-mail: kuri1201@med.kobe-u.ac.jp.

Open access This is an open access article distributed in accordance with the Creative Commons Attribution Non Commercial (CC BY-NC 4.0) license, which permits others to distribute, remix, adapt, build upon this work non-commercially, and license their derivative works on different terms, provided the original work is 
properly cited, appropriate credit is given, any changes made indicated, and the use is non-commercial. See: http://creativecommons.org/licenses/by-nc/4.0/.

\section{ORCID iDs}

Kaori Ueda http://orcid.org/0000-0001-6100-349X

Makoto Nakamura http://orcid.org/0000-0002-6464-4302

\section{REFERENCES}

1 Meyerson C, Van Stavern G, McClelland C. Leber hereditary optic neuropathy: current perspectives. Clin Ophthalmol 2015;9:1165-76.

2 Ueda K, Morizane Y, Shiraga F, et al. Nationwide epidemiological survey of leber hereditary optic neuropathy in Japan. J Epidemiol 2017;27:447-50.

3 Klopstock T, Yu-Wai-Man P, Dimitriadis K, et al. A randomized placebo-controlled trial of idebenone in Leber's hereditary optic neuropathy. Brain 2011;134:2677-86.

4 Yang S, Ma S-Q, Wan X, et al. Long-term outcomes of gene therapy for the treatment of Leber's hereditary optic neuropathy. EBioMedicine 2016;10:258-68.

5 Morimoto T, Miyoshi T, Fujikado T, et al. Electrical stimulation enhances the survival of axotomized retinal ganglion cells in vivo. Neuroreport 2002;13:227-30.

6 Morimoto T, Miyoshi T, Matsuda S, et al. Transcorneal electrical stimulation rescues axotomized retinal ganglion cells by activating endogenous retinal IGF-1 system. Invest Ophthalmol Vis Sci 2005;46:2147-55.

7 Morimoto T, Fujikado T, Choi J-S, et al. Transcorneal electrical stimulation promotes the survival of photoreceptors and preserves retinal function in royal college of surgeons rats. Invest Ophthalmol Vis Sci 2007;48:4725-32.

8 Schatz A, Röck T, Naycheva L, et al. Transcorneal electrical stimulation for patients with retinitis pigmentosa: a prospective, randomized, sham-controlled exploratory study. Invest Ophthalmol Vis Sci 2011;52:4485-96.

9 Gil-Carrasco F, Ochoa-Contreras D, Torres MA, et al. Transpalpebral electrical stimulation as a novel therapeutic approach to decrease intraocular pressure for open-angle glaucoma: a pilot study. $J$ Ophthalmol 2018;2018:1-6.
10 Sabel BA, Hamid AIA, Borrmann C, et al. Transorbital alternating current stimulation modifies BOLD activity in healthy subjects and in a stroke patient with hemianopia: a 7 Tesla fMRI feasibility study. Int J Psychophysiol 2020;154:80-92.

11 Haan N, Song B. Therapeutic application of electric fields in the injured nervous system. Adv Wound Care 2014;3:156-65.

12 Sehic A, Guo S, Cho K-S, et al. Electrical stimulation as a means for improving vision. Am J Pathol 2016;186:2783-97.

13 Kurimoto T, Ueda K, Mori S, et al. A study protocol for evaluating the efficacy and safety of skin electrical stimulation for Leber hereditary optic neuropathy: a single-arm, open-label, nonrandomized prospective exploratory study]]\&gt. Clin Ophthalmol 2019;13:897-904.

14 Kurimoto T, Ueda K, Mori S, et al. A single-arm, prospective, exploratory study to preliminarily test effectiveness and safety of skin electrical stimulation for Leber hereditary optic neuropathy. JCM 2020;9:1359.

15 Tagami Y, Kurimoto T, Miyoshi T, et al. Axonal regeneration induced by repetitive electrical stimulation of crushed optic nerve in adult rats. Jpn J Ophthalmol 2009;53:257-66.

16 Morimoto T, Miyoshi T, Sawai H, et al. Optimal parameters of transcorneal electrical stimulation (Tes) to be neuroprotective of axotomized RGCs in adult rats. Exp Eye Res 2010;90:285-91.

17 Hwang TJ, Karanjia R, Moraes-Filho MN, et al. Natural history of conversion of Leber's hereditary optic neuropathy: a prospective case series. Ophthalmology 2017;124:843-50.

18 Pemp B, Kircher K, Reitner A. Visual function in chronic Leber's hereditary optic neuropathy during idebenone treatment initiated 5 to 50 years after onset. Graefes Arch Clin Exp Ophthalmol 2019;257:2751-7.

19 Perin C, Viganò B, Piscitelli D, et al. Non-invasive current stimulation in vision recovery: a review of the literature. Restor Neurol Neurosci 2020;38:239-50.

20 Tao Y, Chen T, Liu B, et al. The transcorneal electrical stimulation as a novel therapeutic strategy against retinal and optic neuropathy: a review of experimental and clinical trials. Int $J$ Ophthalmol 2016;9:914-9.

21 Liu J, Tong K, Lin Y, et al. Effectiveness of microcurrent stimulation in preserving retinal function of blind leading retinal degeneration and optic neuropathy: a systematic review. Neuromodulation 2021;24:992-1002 